Algebraic 85 Geometric Topology

Volume 2 (2002) 1147-1154

Published: 19 December 2002

ATG

\title{
Equivalences to the triangulation conjecture
}

\author{
DuAne RANDALL
}

\begin{abstract}
We utilize the obstruction theory of Galewski-Matumoto-Stern to derive equivalent formulations of the Triangulation Conjecture. For example, every closed topological manifold $M^{n}$ with $n \geq 5$ can be simplicially triangulated if and only if the two distinct combinatorial triangulations of $R P^{5}$ are simplicially concordant.
\end{abstract}

AMS Classification 57N16, 55S35; 57Q15

Keywords Triangulation, Kirby-Siebenmann class, Bockstein operator, topological manifold

\section{Introduction}

The Triangulation Conjecture (TC) affirms that every closed topological manifold $M^{n}$ of dimension $n \geq 5$ admits a simplicial triangulation. The vanishing of the Kirby-Siebenmann class $K S(M)$ in $H^{4}(M ; Z / 2)$ is both necessary and sufficient for the existence of a combinatorial triangulation of $M^{n}$ for $n \geq 5$ by [7]. A combinatorial triangulation of a closed manifold $M^{n}$ is a simplicial triangulation for which the link of every $i$-simplex is a combinatorial sphere of dimension $n-i-1$. Galewski and Stern [3, Theorem 5] and Matumoto [8] independently proved that a closed connected topological manifold $M^{n}$ with $n \geq 5$ is simplicially triangulable if and only if

$$
\delta_{\alpha} K S(M)=0 \quad \text { in } \quad H^{5}(M ; \operatorname{ker} \alpha)
$$

where $\delta_{\alpha}$ denotes the Bockstein operator associated to the exact sequence $0 \rightarrow \operatorname{ker} \alpha \rightarrow \theta_{3} \stackrel{\alpha}{\longrightarrow} Z / 2 \rightarrow 0$ of abelian groups. Moreover, the Triangulation Conjecture is true if and only if this exact sequence splits by [3] or [11, page 26]. The Rochlin invariant morphism $\alpha$ is defined on the homology bordism group $\theta_{3}$ of oriented homology 3 -spheres modulo those which bound acyclic compact $P L$ 4-manifolds. Fintushel and Stern [1] and Furuta [2] proved that $\theta_{3}$ is infinitely generated.

We freely employ the notation and information given in Ranicki's excellent exposition [11]. The relative boundary version of the Galewski-Matumoto-Stern 
obstruction theory in [11] produces the following result. Given any homeomorphism $f:|K| \rightarrow|L|$ of the polyhedra of closed $m$-dimensional $P L$ manifolds $K$ and $L$ with $m \geq 5, f$ is homotopic to a $P L$ homeomorphism if and only if $K S(f)$ vanishes in $H^{3}(L ; Z / 2)$. More generally, a homeomorphism $f:|K| \rightarrow|L|$ is homotopic to a $P L$ map $F: K \rightarrow L$ with acyclic point inverses if and only if

$$
\delta_{\alpha}(K S(f))=0 \quad \text { in } H^{4}(L ; \operatorname{ker} \alpha) .
$$

Concordance classes of simplicial triangulations on $M^{n}$ for $n \geq 5$ correspond bijectively to vertical homotopy classes of liftings of the stable topological tangent bundle $\tau: M \rightarrow$ BTOP to $B H$ by [3, Theorem 1] and so are enumerated by $H^{4}(M ; \operatorname{ker} \alpha)$. The classifying space $B H$ for the stable bundle theory associated to combinatorial homology manifolds in [11] is denoted by BTRI in [3] and by BHML in [8]. We employ obstruction theory to derive some known and new results and generalizations of [4] and [13] on the existence of simplicial triangulations in section 2 and to record some equivalent formulations of $T C$ in section 3. Although some of these formulations may be known, they do not seem to be documented in the literature.

\section{Simplicial Triangulations}

Let $\delta^{*}$ denote the integral Bockstein operator associated to the exact sequence $0 \rightarrow Z \stackrel{\times 2}{\longrightarrow} Z \stackrel{\rho}{\longrightarrow} Z / 2 \rightarrow 0$. We proceed to derive some consequences of the vanishing of $\delta^{*}$ on Kirby-Siebenmann classes. The coefficient group for cohomology is understood to be $Z / 2$ whenever omitted. Matumoto knew in [8] that the vanishing of $\delta^{*} K S(M)$ implied the vanishing of $\delta_{\alpha} K S(M)$. Let $\iota_{m}$ denote the fundamental class of the Eilenberg-MacLane space $K(Z, m)$. Since $H^{m+1}(K(Z, m) ; G)=0$ for all coefficient groups $G$, trivially $\delta_{\alpha}\left(\rho \iota_{m}\right)=0$ in $H^{m+1}(K(Z, m) ; \operatorname{ker} \alpha)$. Thus $\delta_{\alpha}$ vanishes on $K S(M)$ in (1.1) or $K S(f)$ in (1.2) whenever $\delta^{*}$ does. This observation together with (1.1) and (1.2) justifies the following well-known statements. Every closed connected topological manifold $M^{n}$ with $n \geq 5$ and $\delta^{*} K S(M)=0$ admits a simplicial triangulation. Let $f:|K| \rightarrow|L|$ be any homeomorphism of the polyhedra of closed $m$-dimensional $P L$ manifolds $K$ and $L$ with $m \geq 5$. If $\delta^{*} K S(f)=0$, then $f$ is homotopic to a $P L$ map $F: K \rightarrow L$ with acyclic point inverses.

Proposition 2.1 All $k$-fold Cartesian products of closed 4-manifolds are simplicially triangulable for $k \geq 2$. All products $M^{4} \times S^{1}$ with non-orientable closed 
4-manifolds $M^{4}$ are simplicially triangulable. Let $N^{4}$ be any simply connected closed 4-manifold with $K S(N)$ trivial and also $b=\operatorname{rank}$ of $H_{2}(N ; Z) \geq 1$. Let $f:|K| \rightarrow|L|$ be any homeomorphism with $K S(f)$ nontrivial and $|K|=|L|=$ $N \times S^{1}$. Then $f$ is homotopic to a $P L$ map $F: K \rightarrow L$ with acyclic point inverses.

Proof of 2.1 Since $K S(\gamma)$ is a primitive cohomology class for the universal bundle $\gamma$ on BTOP, we have $K S\left(M_{1} \times M_{2}\right)=K S\left(M_{1}\right) \otimes 1+1 \otimes K S\left(M_{2}\right)$ in $H^{4}\left(M_{1} \times M_{2}\right)$. Triviality of $\delta_{\alpha}$ on $H^{4}\left(M^{4}\right)$ by dimensionality yields triangulability of all $k$-fold products of closed 4-manifolds for $k \geq 2$, and of $M^{4} \times S^{1}$ by (1.1).

The product $N^{4} \times S^{1}$ admits $2^{b}$ distinct combinatorial structures by [7]; moreover, for every non-zero class $u$ in $H^{3}\left(N \times S^{1}\right)$, there is a homeomorphism of polyhedra with distinct combinatorial structures whose Casson-Sullivan invariant is $u$ by [11, page 15]. The vanishing of $\delta^{*} K S(f)$ follows from the triviality of $\delta^{*}$ on $H^{3}\left(N \times S^{1}\right)=\rho\left(H^{2}(N ; Z) \otimes H^{1}\left(S^{1} ; Z\right)\right)$.

No closed 4-manifold $M^{4}$ with $K S(M)$ non-zero can be simplicially triangulated. Yet $k$-fold products of such manifolds $M^{4}$ by (2.1) and their products with spheres or tori produce infinitely many distinct non-combinatorial, yet simplicially triangulable closed manifolds in every dimension $\geq 5$. In contrast, there are no known examples of non-smoothable closed 4-manifolds which can be simplicially triangulated, according to Problem 4.72 of [6, page 287].

Theorem 2.2 Let $M^{n}$ be any closed connected topological manifold with $n \geq 5$ such that the stable spherical fibration determined by the tangent bundle $\tau(M)$ has odd order in $[M, B S G]$. Suppose that either $H_{2}(M ; Z)$ has no 2torsion or else all 2 -torsion in $H_{4}(M ; Z)$ has order 2 . Then $M$ is simplicially triangulable.

Proof The Stiefel-Whitney classes of $M$ are trivial by the hypothesis of odd order. We first consider the special case that $\tau(M)$ is stably fiber homotopically trivial. Let $g: M \rightarrow S G /$ STOP be any lifting of a classifying map $\tau(M): M \rightarrow$ BSTOP in the fibration

$$
S G / \mathrm{STOP} \stackrel{\mathrm{j}}{\longrightarrow} \mathrm{BSTOP} \stackrel{\pi}{\longrightarrow} \mathrm{BSG}
$$

The Postnikov 4-stage of $S G / \mathrm{STOP}$ is $K(Z / 2,2) \times K(Z, 4)$. Now $j^{*} K S(\widetilde{\gamma})=$ $\iota_{2}^{2}+\rho\left(\iota_{4}\right)$ by Theorem 15.1 of $[7$, page 328] where $\widetilde{\gamma}$ denotes the universal bundle over BSTOP. Clearly $\delta^{*}\left(j^{*} K S(\widetilde{\gamma})\right)=\delta^{*}\left(\iota_{2}^{2}\right)=2 u$ where $u$ generates $H^{5}(K(Z / 2,2) ; Z) \approx Z / 4$. If all nonzero 2 -torsion in $H_{4}(M ; Z)$ has order 2 , 
then $\delta^{*} K S(M)=2 g^{*} u=0$. If $H_{2}(M ; Z)$ has no 2-torsion, then $\delta^{*}\left(g^{*} \iota_{2}\right)=0$ so again $\delta^{*} K S(M)=0$. Thus $\delta_{\alpha} K S(M)=0$.

We suppose now that the stable spherical fibration of $\tau(M)$ has order $2 a+1$ in $[M, B S G]$ with $a \geq 1$. Let $s: M \rightarrow S(2 a \tau(M))$ be a section to the sphere bundle projection $p: S(2 a \tau(M)) \rightarrow M$ associated to $2 a \tau(M)$. Now $S(2 a \tau(M))$ is a stably fiber homotopically trivial manifold, since its stable tangent bundle is $(2 a+1) p^{*} \tau(M)$. Since $K S(M)=(2 a+1) K S(M)=s^{*}(K S(S(2 a \tau(M))))$ we conclude that

$$
\delta^{*} K S(M)=s^{*}\left(\delta^{*} K S(S(2 a \tau(M)))\right)=s^{*} 0=0 .
$$

We consider the following homotopy commutative diagram of principal fibrations.

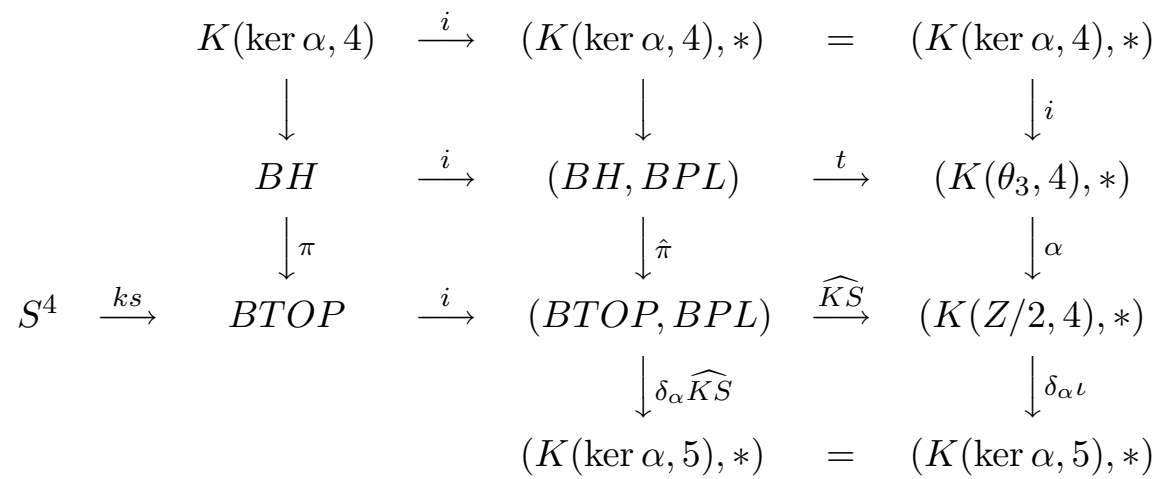

The fiber map $\alpha$ is induced from the path-loop fibration on $K(\operatorname{ker} \alpha, 5)$ via the Bockstein operator $\delta_{\alpha} \iota$ on the fundamental class $\iota$ of $K(Z / 2,4)$. The induced morphism $\alpha_{*}$ on $\pi_{4}$ is the Rochlin morphism $\alpha: \theta_{3} \rightarrow Z / 2$ by construction. The relative principal fibration $\hat{\pi}$ is induced from $\alpha$ via the map $\widehat{K S}$ classifying the relative universal Kirby-Siebenmann class. Thus $(\widehat{K S} \circ i)^{*} \iota=K S(\gamma)$. Inclusion maps are denoted by $i$ in (2.5). The induced morphisms $t_{*}$ and $(\widehat{K S})_{*}$ are isomorphisms on $\pi_{4}$. We employ $(2.5)$ in the proof of Theorem 3.1.

\section{Equivalent formulations to $T C$}

Galewski and Stern constructed a non-orientable closed connected 5-manifold $M^{5}$ in [4] such that $S q^{1} K S(M)$ generates $H^{5}(M) \approx Z / 2$. They also proved that any such $M^{5}$ is "universal" for TC. Moreover, Theorem 2.1 of [4] essentially affirms that either $T C$ is true or else no closed connected topological $n$-manifold $M^{n}$ with $S q^{1} K S(M) \neq 0$ and $n \geq 5$ can be simplicially triangulated. 


\section{Theorem 3.1}

The following statements are equivalent to the Triangulation Conjecture.

(1) Any (equivalently all) of the classes $\delta_{\alpha} K S(\gamma), \delta_{\alpha} \widehat{K S}$, and $\delta_{\alpha} \iota$ in (2.5) is trivial if and only if any (equivalently all) of the fiber maps $\pi, \hat{\pi}$, and $\alpha$ in (2.5) admits a section.

(2) The essential map $f: S^{4} \cup_{2} e^{5} \rightarrow$ BTOP lifts to $B H$ in (2.5).

(3) $S q^{1} K S(\hat{\gamma}) \neq 0$ in $H^{5}(B H)$ for the universal bundle $\hat{\gamma}=\pi^{*} \gamma$ on $B H$.

(4) Any closed connected topological manifold $M^{n}$ with $S q^{1} K S(M) \neq 0$ and $n \geq 5$ admits a simplicial triangulation.

(5) Every homeomorphism $f:|K| \rightarrow|L|$ with $K S(f)$ non-trivial is homotopic to a $P L$ map with acyclic point inverses where $K$ and $L$ are any combinatorially distinct polyhedra with $|K|=|L|=N^{4} \times R P^{2}$. Here $N^{4}$ denotes any simply connected, closed 4-manifold with $K S(N)$ trivial and positive rank for $\mathrm{H}_{2}(N ; Z)$.

(6) All combinatorial triangulations of each closed connected PL manifold $M^{n}$ with $n \geq 5$ are concordant as simplicial triangulations.

(7) The two distinct combinatorial triangulations of $R P^{5}$ are simplicially concordant.

(8) Every closed connected topological manifold $M^{n}$ with $n \geq 5$ that is stably fiber homotopically trivial admits a simplicial triangulation.

Proof $T C \Leftrightarrow(1)$ Statement (1) is equivalent to the splitting of the exact sequence $0 \rightarrow \operatorname{ker} \alpha \rightarrow \theta_{3} \stackrel{\alpha}{\longrightarrow} Z / 2 \rightarrow 0$ through the induced morphisms on homotopy in dimension 4 .

$T C \Leftrightarrow(2)$ Let $k s: S^{4} \rightarrow$ BTOP represent the Kirby-Siebenmann class in homotopy. That is, $[k s]$ has order 2 and is dual to $K S(\gamma)$ under the mod 2 Hurewicz morphism. Now $k s$ admits an extension $f: S^{4} \cup_{2} e^{5} \rightarrow$ BTOP, since the cofibration exact sequence

$$
\pi_{5}(\mathrm{BTOP}) \longrightarrow\left[\mathrm{S}^{4} \cup_{2} \mathrm{e}^{5}, \mathrm{BTOP}\right] \rightarrow \pi_{4}(\mathrm{BTOP}) \stackrel{\times 2}{\longrightarrow} \pi_{4}(\mathrm{BTOP})
$$

corresponds to $0 \longrightarrow Z / 2 \longrightarrow Z \oplus Z / 2 \stackrel{\times 2}{\longrightarrow} Z \oplus Z / 2$. If $g: S^{4} \cup_{2} e^{5} \rightarrow B H$ is any lifting of $f$, the composite map using (2.5)

$$
h: S^{4} \subset S^{4} \cup_{2} e^{5} \stackrel{g}{\longrightarrow} B H \stackrel{i}{\longrightarrow}(B H, B P L) \stackrel{t}{\longrightarrow}\left(K\left(\theta_{3}, 4\right), *\right)
$$

produces $u=[h]$ in $\theta_{3}$ with $2 u=0$ and $\alpha(u)=1$, since $\alpha(u)=[\alpha \circ h]=$ $[\widehat{K S} \circ k s]$ generates $\pi_{4}(K(Z / 2,4))$. Thus $T C$ is true. Conversely, if $T C$ is true, a section $s:$ BTOP $\rightarrow$ BH to $\pi$ in (2.5) gives a lifting $s \circ f$ of $f$. 
$T C \Leftrightarrow(3)$ Properties of $K S(\gamma)$ are enumerated in [9] and [10]. Since $S q^{1} K S(\gamma) \neq 0$, a section $s$ to $\pi$ in $(2.5)$ gives $S q^{1}(K S(\hat{\gamma}) \neq 0$ so $T C$ implies 3 . We now assume that $T C$ is false and claim that the generator $S q^{1} \iota$ for $H^{5}(K(Z / 2,4)) \approx Z / 2$ lies in the image of

$$
H^{5}(K(\operatorname{ker} \alpha, 5)) \approx H o m\left(\pi_{5}\left(K\left(\operatorname{ker}_{\alpha}, 5\right)\right), Z / 2\right) \approx H o m(\operatorname{ker} \alpha, Z / 2) .
$$

The Serre exact sequence then gives $\alpha^{*}\left(S q^{1} \iota\right)=0$ in $H^{5}\left(K\left(\theta_{3}, 4\right)\right)$ so

$$
S q^{1} K S(\hat{\gamma})=(t \circ i)^{*}\left(\alpha^{*} S q^{1} \iota\right)=0 .
$$

Thus we must construct a morphism ker $\alpha \rightarrow Z / 2$ which does not extend to $\theta_{3}$. We consider the sequence $\operatorname{ker} \alpha \stackrel{\times 2}{\longrightarrow} \operatorname{ker} \alpha \stackrel{\rho}{\longrightarrow} \operatorname{ker} \alpha \otimes Z / 2$ and define $h:$ ker $\alpha \otimes Z / 2 \rightarrow Z / 2$ as follows. $h(v)=1$ if and only if $v=\rho(2 z)$ for some $z \in \theta_{3}$ with $\alpha(z)=1$. Now $h$ is a well-defined and non-trivial morphism, since $\theta_{3}$ does not have an element $u$ with $2 u=0$ and $\alpha(u)=1$ by hypothesis. The composite morphism $h \circ \rho: \operatorname{ker} \alpha \rightarrow Z / 2$ does not extend to $\theta_{3}$.

$T C \Leftrightarrow(4)$ Suppose $M^{n}$ with $S q^{1} K S(M) \neq 0$ admits a simplicial triangulation. Now $S q^{1} K S(M)=g^{*} S q^{1} K S(\hat{\gamma})$ for any lifting $g: M \rightarrow B H$ of $\tau: M \rightarrow$ BTOP. Since $S q^{1} K S(\hat{\gamma}) \neq 0, T C$ holds by (3).

$T C \Leftrightarrow(5) \quad$ Clearly triviality of $\delta_{\alpha} \widehat{K S}$ in $(2.5)$ gives $\delta_{\alpha} K S(f)=0$ via naturality for every $f$. Suppose that $\delta_{\alpha} K S(f)=0$ for any such $f$ in 5 . Now $K S(f)=$ $\rho(v) \otimes i^{*} a$ in $\rho\left(H^{2}(M ; Z)\right) \otimes H^{1}\left(R P^{2}\right) \approx H^{3}(L)$. Here $a$ generates $H^{*}\left(R P^{\infty}\right)$ and $i: R P^{2} \subset R P^{\infty}$. Naturality via the universal example $C P^{\infty} \times R P^{\infty}$ for $\rho(v) \otimes i^{*} a$ gives $\delta_{\alpha} K S(f)=v \otimes \delta_{\alpha}\left(i^{*} a\right)$. Since $i^{*}: H^{2}\left(R P^{\infty} ; \operatorname{ker} \alpha\right) \rightarrow$ $H^{2}\left(R P^{2} ; \operatorname{ker} \alpha\right)$ is a monomorphism, $\delta_{\alpha}\left(i^{*} a\right)=0$ if and only if $\delta_{\alpha}(a)=0$. Now $\delta_{\alpha}(a)=0$ if and only if $T C$ is true via the fibration

$$
K(\operatorname{ker} \alpha, 1) \longrightarrow K\left(\theta_{3}, 1\right) \stackrel{\alpha}{\longrightarrow} R P^{\infty} .
$$

$T C \Leftrightarrow(6) \Leftrightarrow(7) \quad T C$ holds if and only if $\delta_{\alpha} \iota=0$ for the fundamental class $\iota$ of $K(Z / 2,3)$. Concordance classes of simplicial triangulations of $M^{n}$ arising from combinatorial triangulations differ by classes in $\delta_{\alpha} H^{3}(M)$. This subgroup of $H^{4}(M, \operatorname{ker} \alpha)$ is trivial by naturality if $\delta_{\alpha} \iota=0$. Conversely, $\delta_{\alpha} H^{3}\left(R P^{5}\right)=0$ if the two distinct combinatorial triangulations of $R P^{5}$ given by Theorem 16.5 in [7, pages 332 and 337] are simplicially concordant. But $\delta_{\alpha}\left(a^{3}\right)=0$ if and only if $\delta_{\alpha} \iota=0$ via the skeletal inclusion $R P_{3}^{5} \subset K(Z / 2,3)$ and naturality for $R P^{5} \rightarrow R P_{3}^{5}$.

$T C \Leftrightarrow(8)$ Similar to Theorem 5.1 of [12], we consider a regular neighborhood of the 9-skeleton of $S G /$ STOP embedded in $R^{m}$ for some $m \geq 19$ in order 
to obtain a smoothly parallelizable manifold $W$ with boundary and a map $g: W \rightarrow S G /$ STOP which is a homotopy equivalence through dimension 7 . The double $D W$ is smoothly parallelizable and admits an extension $\widehat{g}: D W \rightarrow$ $S G /$ STOP. Note that $(\widehat{g})^{*}$ is a monomorphism through dimension 7 . Let $h$ : $M \rightarrow D W$ be a degree one normal map. Now $M$ is stably fiber homotopically trivial and $h^{*}$ is a monomorphism in cohomology. In particular, $(\widehat{g} \circ h)^{*}$ is a monomorphism on $H^{5}(S G / \mathrm{STOP} ; \operatorname{ker} \alpha)$. We conclude that $\delta_{\alpha} K S(M)=$ $(\widehat{g} \circ h)^{*}\left(\delta_{\alpha} \iota_{2}^{2}\right)=0$ if and only if $\delta_{\alpha} \iota_{2}^{2}=0$ for the fundamental class $\iota_{2}$ of $K(Z / 2,2)$. So statement (8) yields $\delta_{\alpha} \iota_{2}^{2}=0$.

Let $f: K(Z / 2,2) \rightarrow K(Z / 2,4)$ classify $\iota_{2}^{2}$. Since $\delta_{\alpha} \iota_{2}^{2}=0$ assuming statement (8), $f$ admits a lifting $h: K(Z / 2,2) \rightarrow K\left(\theta_{3}, 4\right)$ in $(2.5)$ such that $f=\alpha \circ h$. The diagram

$$
\begin{array}{lcccc} 
& {\left[C P^{3}, K\left(\theta_{3}, 4\right)\right]} & \approx & \theta_{3} \\
& h_{*} & \downarrow \alpha_{*} & & \downarrow \\
Z / 2 \approx\left[C P^{3}, K(Z / 2,2)\right] & \stackrel{f_{*}}{\longrightarrow} & {\left[C P^{3}, K(Z / 2,4)\right]} & \approx & Z / 2
\end{array}
$$

yields a splitting to the exact sequence $0 \rightarrow \operatorname{ker} \alpha \rightarrow \theta_{3} \rightarrow Z / 2 \rightarrow 0$ so TC holds.

\section{References}

[1] R Fintushel, R J Stern, Instanton homology of Seifert fibred homology three spheres, Proc. London Math. Soc. 61 (1990) 109-137

[2] M Furuta, Homology cobordism group of homology 3-spheres, Inventiones Math. 100 (1990) 339-355

[3] D E Galewski, R J Stern, Classification of simplicial triangulations of topological manifolds, Annals of Math. 111 (1980) 1-34

[4] DE Galewski, RJ Stern, A universal 5-manifold with respect to simplicial triangulations, from: "Geometric Topology (Proc. Georgia Topology Conf. Athens, Ga. 1977)", Academic Press (1979) 345-350

[5] D E Galewski, R J Stern, Simplicial triangulations of topological manifolds, from: "Algebraic and Geometric Topology (Stanford, Calif. 1976) Part 2", A.M.S. Proc. of Symposia in Pure Mathematics, Vol. 32 (1978) 7-12

[6] R Kirby, Problems in low-dimensional topology, from: "Geometric Topology (Athens, GA, 1993)", AMS/IP Studies in Advanced Math. 2.2 (1997) 35-473

[7] R Kirby, L Siebenmann, Foundational essays on topological manifolds, smoothings, and triangulations, Annals of Math. Studies 88, Princeton Univ. Press, Princeton (1977) 
[8] T Matumoto, Triangulation of manifolds, from: "Algebraic and Geometric Topology (Stanford, Calif. 1976) Part 2", A.M.S. Proc. of Symposia in Pure Mathematics, Vol. 32 (1978) 3-6

[9] R J Milgram, Some remarks on the Kirby-Siebenmann class, from: "Göttingen Conference on Algebraic Topology and Transformation Groups", Lecture Notes in Math. No. 1361, Springer-Verlag (1988) 247-252

[10] D Randall, On 4-dimensional bundle theories, from: "Differential Topology, Foliations and Group Actions", Contemp. Math. Vol. 161, Amer. Math. Soc. (1994) 217-233

[11] A A Ranicki, On the Hauptvermutung, from: "The Hauptvermutung Book", K-Monographs in Math., Kluwer Academic Publishers (1996) 3-31

[12] Y B Rudyak, On Thom Spectra, Orientability, and Cobordism, Monographs in Math., Springer-Verlag (1998)

[13] L. Siebenmann, Are non-triangulable manifolds triangulable? from: "Topology of Manifolds, Proc. 1969 Georgia Topology Conference", Markham Press (1970) $77-84$

Department of Mathematics and Computer Science

Loyola University, New Orleans, LA 70118, USA

Email: randall@loyno.edu

Received: 19 July 2002 